\title{
Septic Pulmonary Embolism Case Report: Optimal Outcome after Insertion of an Inferior Vena Cava Filter in a Patient with Staphylococcus aureus Bacteraemia
}

\author{
Isabel Esteves, ${ }^{1}$ Sofia Vidal Castro, ${ }^{1}$ Francisco Abecasis, ${ }^{2}$ Cristina Camilo, ${ }^{2}$ Marisa Vieira, ${ }^{2}$ \\ Dinis da Gama, ${ }^{3}$ and Manuela Correia ${ }^{2}$ \\ ${ }^{1}$ Child and Family Department, Santa Maria Hospital, Intensive Care Unit, Avenida Professor Egas Moniz, 1649-035 Lisbon, Portugal \\ ${ }^{2}$ Paediatric Intensive Care Unit, Child and Family Department, Santa Maria Hospital, Lisbon Medical Faculty, Lisbon, Portugal \\ ${ }^{3}$ Vascular Surgery Department, Santa Maria Hospital, Lisbon Medical Faculty, Lisbon, Portugal
}

Correspondence should be addressed to Isabel Esteves, isabel.castro.menezes@gmail.com

Received 25 October 2009; Revised 23 February 2010; Accepted 31 March 2010

Academic Editor: Stefan Burdach

Copyright (C 2010 Isabel Esteves et al. This is an open access article distributed under the Creative Commons Attribution License, which permits unrestricted use, distribution, and reproduction in any medium, provided the original work is properly cited.

\begin{abstract}
A 14-year-old patient presented with bilateral pneumonia and pleural effusions, septic arthritis of the hip, deep venous thrombosis, and pulmonary thromboembolism. Methicillin-sensitive Staphylococcus aureus (S. aureus) containing the Panton Valentine Leukocidin (PVL) genes was isolated. Contraindication to anticoagulation prompted inferior vena cava filter placement. He completed 4 weeks of treatment with flucloxacillin, with good clinical outcome. S. aureus containing PVL genes should be sought in cases of necrotizing pneumonia as it seems to increase the risk of severe multifocal infection and thrombotic complications. There are few reports of placement of filters during S. aureus sepsis and bacteraemia. This case highlights that when anticoagulation is not feasible, an inferior vena cava filter can be inserted safely, even in patients with active sepsis and high risk for seeding of the filter. Long-term follow-up confirmed a successful outcome with sterilization of the septic thrombosis with no further pulmonary embolism or additional sepsis episodes.
\end{abstract}

\section{Introduction}

Staphylococcus aureus (S. aureus) may cause communityacquired multifocal severe disease on otherwise healthy children and adolescents. Multiple virulence factors have been identified as important to the pathogenesis of infection [1]. One of those factors is Panton-Valentine Leukocidin (PVL), an exotoxin that destroys leukocytes by pore-forming activity. Several studies have shown an association of $S$. aureus containing PVL genes with a more severe inflammatory response $[2,3]$ and clinically with necrotic skin lesions and abscesses [4], arthritis/osteomyelitis complicated by septic deep venous thrombosis $[3,5]$ and severe necrotizing pneumonia [6]. However, the role of PVL in the pathogenesis of community-acquired infections, especially in methicillinresistant strains, is still under discussion $[1,7,8]$.

The management of major deep venous thrombosis in septic patients may be difficult. The high risk for complications such as worsening sepsis, metastatic infection, and pulmonary embolism, is an important argument to consider placement of an inferior vena cava (IVC) filter. Those complications also raise questions about the safety and long-term effectiveness of central venous filters, inserted during active sepsis and bacteraemia. Greenfield and Proctor [9] reviewed 175 septic patients who received intravascular filters and found no reports of patients with recurrent sepsis episodes or any significant adverse events. On the other hand, an IVC filter has been shown to become infected during active sepsis [10].

Considering these issues, the authors report a case of safe and effective insertion of an IVC filter in a patient with septic embolism with good long-term outcome.

\section{Case Report}

A 14-year-old male adolescent had a toe trauma complicated with local soft tissue infection and necrosis. Ten days later 
he presented with fever, cough, thoracic and right inguinal pain. The physical exam revealed signs of minor respiratory distress, $97 \%$ oxygen saturation on room air, decreased breath sounds on the right, inflammatory signs of the right lower leg, and a toe wound. Laboratory evaluation at admission showed: leukocyte count $18.2 \times 10^{9} / \mathrm{L}$ with $91 \%$ neutrophils, haemoglobin $1.72 \mathrm{mmol} / \mathrm{L}$, platelets $114 \times 10^{9} / \mathrm{L}$, and C-reactive protein $250 \mathrm{mg} / \mathrm{L}$. The chest radiograph was consistent with bilateral pneumonia and minor pleural effusions. The venous doppler ultrasound confirmed a deep venous thrombosis (DVT) of the right femoral vein. Blood cultures were performed and the patient was started on intravenous antibiotic therapy with piperacillin-tazobactam and subcutaneous low-molecular weight heparin. The transthoracic echocardiogram revealed a good ventricular function and no signs of cardiac vegetations.

On the third day after admission, thoracic pain and respiratory distress increased. Considering the hypothesis of septic pulmonary thromboembolism (PT) a chest computed tomography (CT) was performed, showing bilateral thoracic infiltrates and pleural effusion. The diagnostic thoracocentesis revealed a pleural empyema.

Twelve hours later, the patient developed signs of shock and was admitted to the paediatric intensive care unit (PICU). Abdominal ultrasound revealed haemoperitoneum and hepatic hematoma probably due to hepatic puncture during thoracocentesis. Anticoagulation was stopped. A second CT scan showed signs of pulmonary thromboembolism and right hip fluid collection suggestive of septic arthritis. Doppler studies of inferior vena cava and ileo-femoral vessels identified a large thrombus in the right iliac veins. The case was discussed with the vascular surgeons and a low-profile percutaneously introducible IVC filter (titanium Greenfield filter, Medi-tech, Watertown, MA) was placed (Figure 1). Simultaneously, right hip arthrotomy with debridment and drainage was performed.

Methicillin-sensitive S. aureus was isolated from blood cultures and synovial fluid (Figure 2(a)) prompting antibiotic therapy change to flucloxacillin. PVL genes (lukS-PV and lukF-PV) were detected on polymerase chain reaction (PCR) assays (Figure 2(b)). Pleural fluid had no microbiologic isolation.

The patient completed four weeks of intravenous flucloxacillin with good clinical outcome, being afebrile since the sixth day of antibiotic therapy. From a pulmonary standpoint, the patient was stabilized two days after the IVC filter placement, with no other respiratory distress or thoracic pain episodes. Repeated abdominal ultrasounds in the first month after admission showed improvement of haemoperitoneum.

Immunological studies were performed two months after admission, when the patient had completely recovered. Immunodeficiency was ruled out by normal levels of immunoglobulins and phagocyte oxidative capacity (dihydrorhodamine assay).

The evaluation for venous thrombosis risk factors revealed a common genetic polymorphism of the enzyme methylenetetrahydrofolate reductase (MTHFR) with heterozygosity for $677 \mathrm{C}>\mathrm{T}$ substitution and another heterozy-

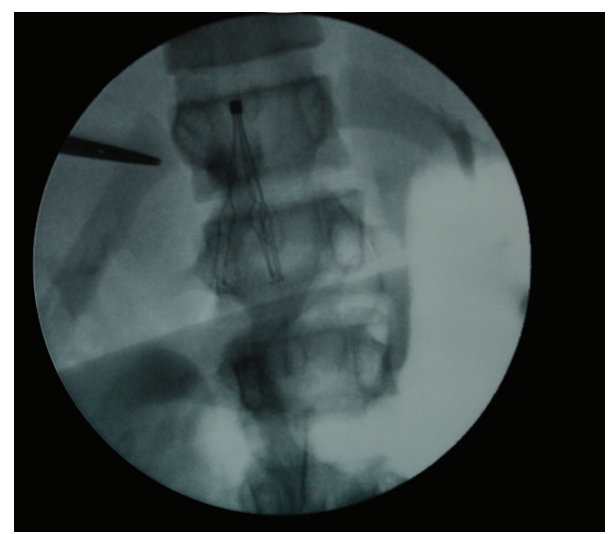

FIGURE 1: Intraoperative angiography showing inferior vena cava (IVC) filter placement.

gous polymorphism of plasminogen activator inhibitor 1 (PAI-1) locus with the allelic variant 675G $>A$ (4G/5G), both tested with $P C R$ assays. Other diagnostic work-up for hypercoagulability yielded normal results (prothrombin time, activated partial thromboplastin time; antiphospholipid antibodies; levels of fibrinogen, antithrombin III, protein $\mathrm{C}$, protein $\mathrm{S}$; testing for factor $\mathrm{V}$ Leiden mutation, and prothrombin G20210A mutation).

At 14 months of follow-up there were no other episodes of fever or possible bacteraemia, IVC filter migration, progressive IVC thrombosis, pulmonary embolism, or significant hip joint dysfunction.

\section{Discussion}

It is known that $S$. aureus strains harbouring the PVL genes are associated with complicated skin and soft tissue infections $[3,5,6]$. PVL genes are also implicated in bone and joint infections with a higher rate of deep venous thrombosis $[2,5]$. These findings are in accordance with the severity of the community-acquired soft tissue infection in our previously healthy patient, complicated with right hip septic arthritis, right femoral venous thrombosis, septic PT, and bilateral pneumonia. In a previous study, the occurrence of septic PT in children infected with a $S$. aureus strain containing PVL genes, represented 19\% of the abnormal pulmonary findings in these patients [6]. Deep venous thrombosis should always be considered in patients who present with a musculoskeletal infection and respiratory symptoms, especially if a staphylococcal infection is possible. Also an echocardiography should be routinely performed in this clinical scenario, in spite of conflicting evidence on the importance of isolated $S$. aureus bacteraemia and the risk of endocarditis $[6,11,12]$.

In the reported case the diagnostic work-up included routine evaluation tests for immunodeficiency and thrombophilia. In spite of the known aggressiveness of $S$. aureus strains harbouring the PVL genes, we believe that hereditary host susceptibility for infection or thrombosis should be 


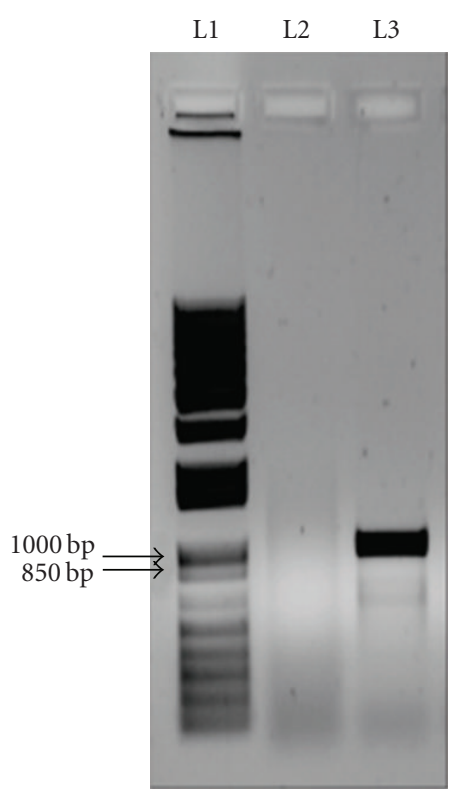

(a)

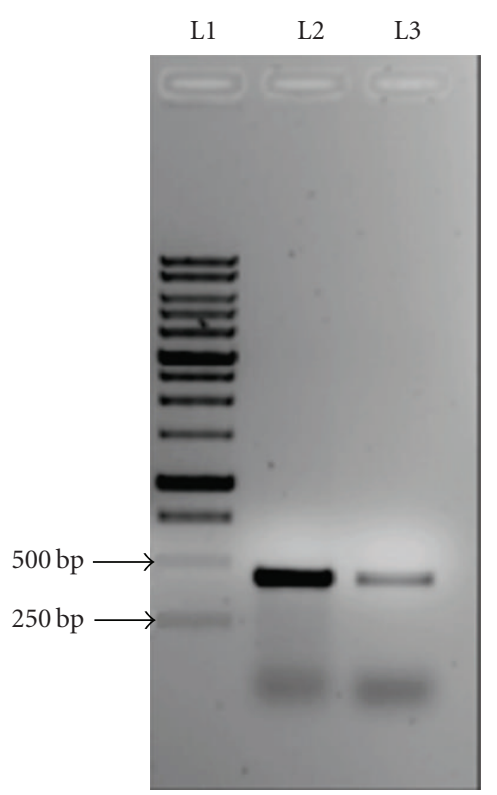

(b)

FIgure 2: PCR amplification of mecA gene products (a) and the genes that code PVL (b) visualized on gel electrophoresis. (a) Lanes (L): L1-mecA gene PCR kit; L2_Patient; L3—Positive Control. (b) Lanes (L): L1—PVL gene PCR kit; L2—Patient; L3—Positive Control.

sought, especially in young children or whenever there is a family history. Our patient had two common polymorphisms of the MTHFR and PAI-1 genes. Although their clinical significance as risk factors for thromboembolic events is uncertain, they might play a role when associated with $S$. aureus infections.

When septic PT is diagnosed, prompt antimicrobial and anticoagulation therapy should be instituted [5]. In our patient, the anticoagulation had to be stopped because of active bleeding, so a surgical/mechanical solution was sought and an IVC filter was placed.

The significant morbidity and mortality risk of acute disseminated S. aureus disease after deep vein thrombophlebitis is well recognized [13]. Remarkably, there are few reports of placement of filters during $S$. aureus sepsis and bacteraemia in children. A retrospective review [14] of 15 children 18 years or younger, suggested that long-standing vena cava filters were safe and prevented pulmonary embolism, but none was placed in the setting of infection. The Food and Drug Administration guidance for intravascular filters [15] defends that sepsis and septic embolism are contraindications for IVC filter placement.

An in vivo study of the effectiveness of Greenfield filters in animals with infected thrombus, found that antibiotics successfully sterilized the filter, septic embolus, and vena cava wall [16]. On the contrary, different surgical options used in the past, like vena caval ligation, were associated with pockets of infection and abscess, independently of antibiotic therapy [17].

Gonzalez et al. [5] reviewed 3 patients with filter placement after venous thrombosis in S. aureus osteomyelitis, and found no filter migration, recurrent septic PT or IVC thrombosis.
Greenfield and Proctor [9] reviewed 175 septic patients who received intravascular filters and demonstrated in their study that the placement of filters during sepsis did not add to the mortality and was an alternative when anticoagulation was not feasible. The authors found no clinical reports of patients requiring filter removal secondary to sepsis or any other adverse events related to septicaemia following filter placement. The good results may be associated with the inert materials used in the Greenfield filter (stainless steel and titanium). In this way, only a trapped embolus can become infected, a problem that is easily solved with adequate antibiotic therapy. In spite of the significant number of patients included, these results may be biased by the retrospective analysis and lack of specific outcomes measure, with an important number of drop-outs on followup evaluation (only $32 \%$ of the patients).

Also, one recent report describes IVC filter insertion in a patient with Candida glabrata septic thrombophlebitis that resulted in IVC filter infection [10].

We consider that a severe invasive infection is an important risk factor for venous filter placement, but it seems to be warranted for septic patients who are haemodynamically unstable and cannot tolerate an additional respiratory compromise associated with pulmonary emboli. The indications for this procedure in paediatric sepsis should be reviewed, including significant PT and contraindications for anticoagulation, as in our patient. Risk factors for the development of persistent bacteraemia/fungaemia must also be weighted, such as prolonged intensive care unit stay, other prostetic devices, immunosuppression, and antibiotic resistance.

This case highlights the importance of considering $S$. aureus strains containing the PVL genes as the etiologic agent 
when there are signs of bone, joint, or soft tissue infection associated with severe pneumonia and thromboembolic events. In this setting the placement of an IVC filter may be a safe and effective strategy to prevent PT when anticoagulation is not indicated.

\section{References}

[1] T. Baba, F. Takeuchi, M. Kuroda, et al., "Genome and virulence determinants of high virulence community-acquired MRSA," The Lancet, vol. 359, no. 9320, pp. 1819-1827, 2002.

[2] C. E. Bocchini, K. G. Hulten, E. O. Mason, B. E. Gonzalez, W. A. Hammerman, and S. L. Kaplan, "Panton-Valentine leukocidin genes are associated with enhanced inflammatory response and local disease in acute hematogenous Staphylococcus aureus osteomyelitis in children," Pediatrics, vol. 117, no. 2, pp. 433-440, 2006.

[3] A. Swaminathan, D. Massasso, I. Gotis-Graham, and I. Gosbell, "Fulminant methicillin-sensitive Staphylococcus aureus infection in a healthy adolescent, highlighting "PantonValentine leucocidin syndrome"', Internal Medicine Journal, vol. 36, no. 11, pp. 744-747, 2006.

[4] P. Del Giudice, V. Blanc, A. De Rougemont, et al., "Primary skin abscesses are mainly caused by Panton-Valentine leukocidin-positive Staphylococcus aureus strains," Dermatology, vol. 219, no. 4, pp. 299-302, 2009.

[5] B. E. Gonzalez, J. Teruya, D. H. Mahoney, et al., "Venous thrombosis associated with staphylococcal osteomyelitis in children," Pediatrics, vol. 117, no. 5, pp. 1673-1679, 2006.

[6] B. E. Gonzalez, K. G. Hulten, M. K. Dishop, et al., "Pulmonary manifestations in children with invasive community-acquired Staphylococcus aureus infection," Clinical Infectious Diseases, vol. 41, no. 5, pp. 583-590, 2005.

[7] G. Lina, Y. Piemont, F. Godail-Gamot, et al., "Involvement of Panton-Valentine leukocidin-producing Staphylococcus aureus in primary skin infections and pneumonia," Clinical Infectious Diseases, vol. 29, no. 5, pp. 1128-1132, 1999.

[8] S. Schlebusch, J. M. Schooneveldt, F. Huygens, and G. R. Nimmo, "Prevalence of Staphylococcus aureus strains in an Australian cohort, 1989-2003: evidence for the low prevalence of the toxic shock toxin and Panton-Valentine leukocidin genes," European Journal of Clinical Microbiology and Infectious Diseases, vol. 28, no. 10, pp. 1183-1189, 2009.

[9] L. J. Greenfield and M. C. Proctor, "Vena caval filter use in patients with sepsis: results in 175 patients," Archives of Surgery, vol. 138, no. 11, pp. 1245-1248, 2003.

[10] M. S. Meda, A. J. Lopez, and A. Guyot, "Candida inferior vena cava filter infection and septic thrombophlebitis," The British Journal of Radiology, vol. 80, no. 950, pp. e48-e49, 2007.

[11] J. Abraham, C. Mansour, E. Veledar, B. Khan, and S. Lerakis, "Staphylococcus aureus bacteremia and endocarditis: the Grady Memorial Hospital experience with methicillin-sensitive $S$ aureus and methicillin-resistant $S$. aureus bacteremia," American Heart Journal, vol. 147, no. 3, pp. 536-539, 2004.

[12] B. E. Gonzalez, G. Martinez-Aguilar, K. G. Hulten, et al., "Severe staphylococcal sepsis in adolescents in the era of community-acquired methicillin-resistant Staphylococcus aureus," Pediatrics, vol. 115, no. 3, pp. 642-648, 2005.

[13] A. Gorenstein, E. Gross, S. Houri, G. Gewirts, and S. Katz, "The pivotal role of deep vein thrombophlebitis in the development of acute disseminated staphylococcal disease in children," Pediatrics, vol. 106, no. 6, article E87, 2000.
[14] M. D. Cahn, M. J. Rohrer, M. B. Martella, and B. S. Cutler, "Long-term follow-up of Greenfield inferior vena cava filter placement in children," Journal of Vascular Surgery, vol. 34, no. 5, pp. 820-825, 2001.

[15] Food and Drug Administration, "Guidance for Cardiovascular Filter 510(K) Submissions," Rockville, Md, USA: Center for Devices and Radiological Help, Interventional Cardiology Devices Branch, 1999, http://www.fda.gov/MedicalDevices/ DeviceRegulationandGuidance/GuidanceDocuments/ucm07 3776.htm.

[16] J. H. Scott, C. L. Anderson, and P. S. Shankar, "Septicemia from infected caval "umbrella", Journal of the American Medical Association, vol. 243, no. 11, pp. 1133-1134, 1980.

[17] J. W. R. Peyton, M. B. Hylemon, L. J. Greenfield, S. Crute, and H. Sugerman, "Comparison of Greenfield filter and vena caval ligation for experimental septic thromboembolism," Surgery, vol. 93, pp. 533-537, 1983. 


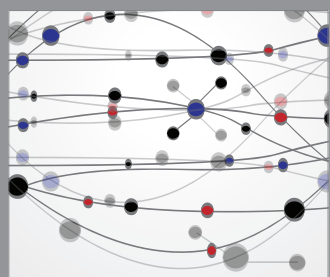

The Scientific World Journal
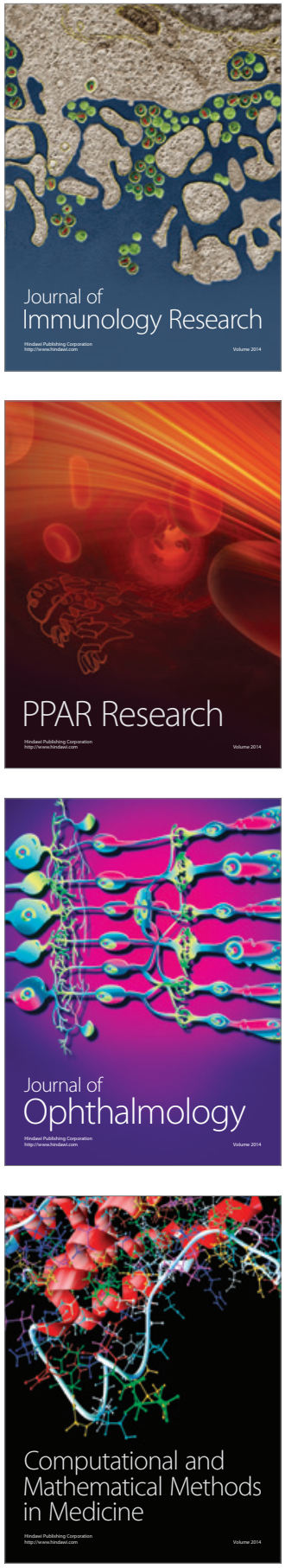

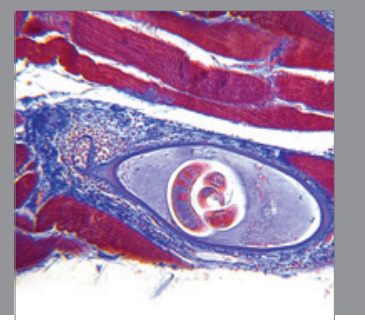

Gastroenterology

Research and Practice
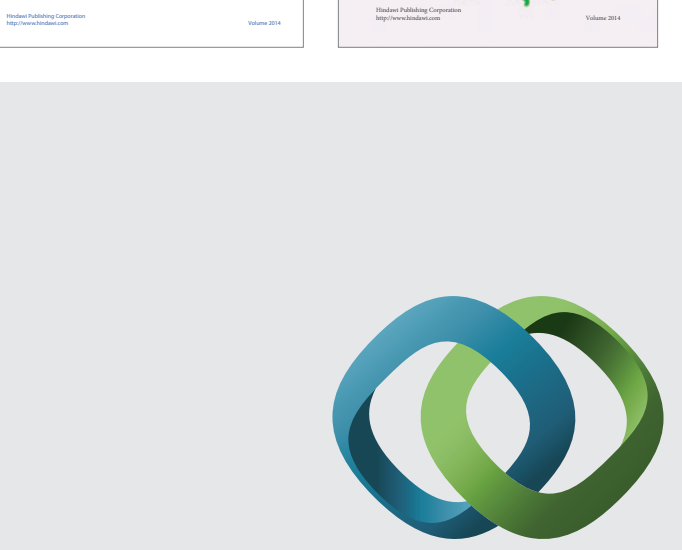

\section{Hindawi}

Submit your manuscripts at

http://www.hindawi.com
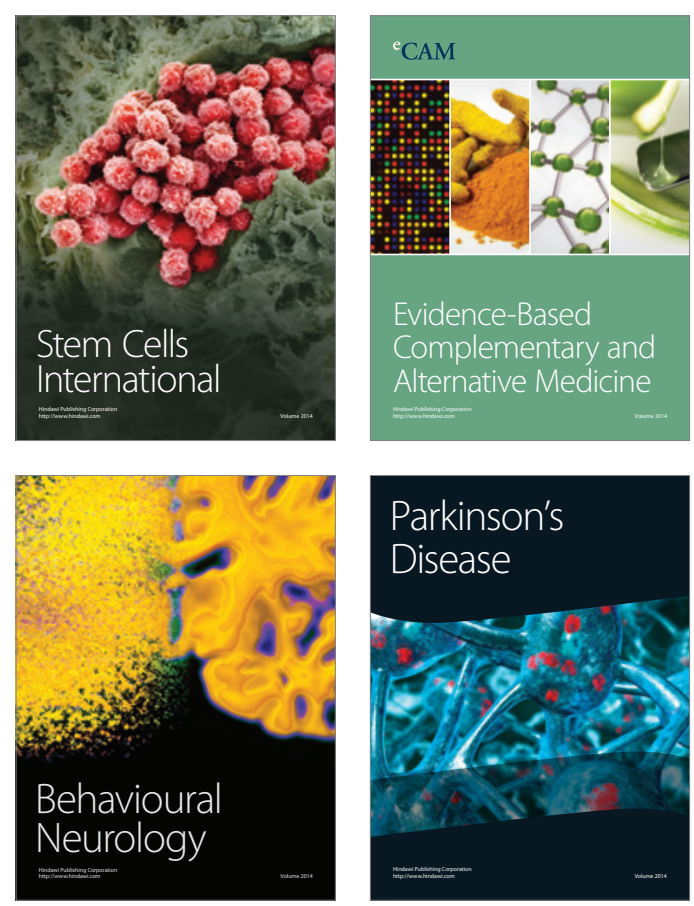

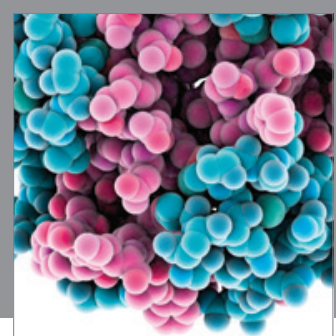

Journal of
Diabetes Research

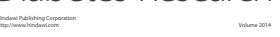

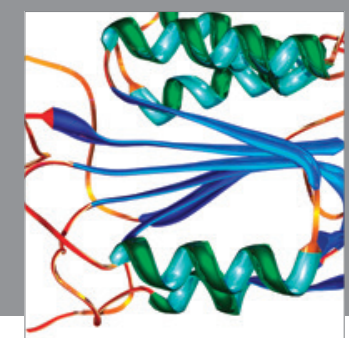

Disease Markers
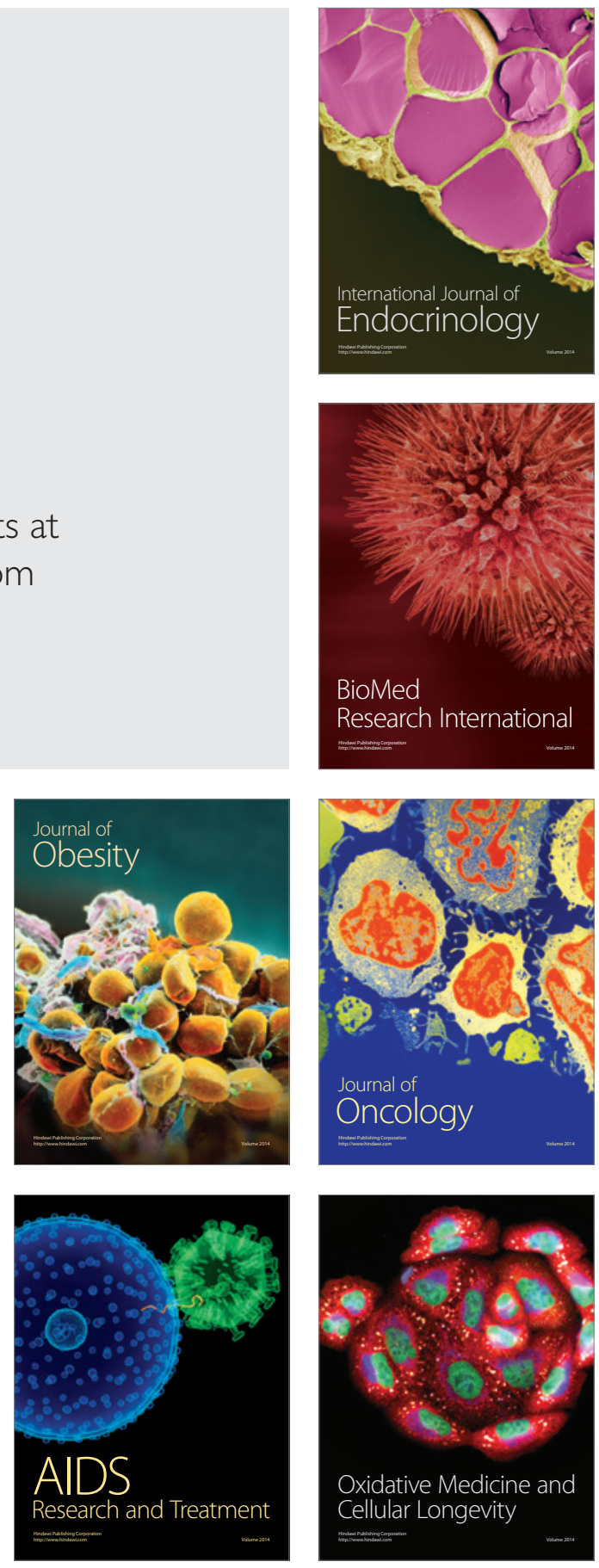